\title{
Commentary: "The Etiology of Down Syndrome" Hultén $\mathrm{MA}^{1 *}$, Iwarsson $\mathrm{E}^{1}$, Jonasson $\mathrm{J}^{2}$ \\ 'Department of Molecular Medicine and Surgery, Center for Molecular Medicine, Karolinska Institutet, Stockholm, Sweden ${ }^{2}$ Department of Clinical and Experimental Medicine, Linköping University, Linköping, Sweden
}

Article Info

\section{Article Notes}

Received: January 3, 2019

Accepted: February 18, 2019

\section{*Correspondence:}

Dr. Maj Anita Hultén, Prof em Clinical Genetics, Sweden; Email: maj.hulten@ki.se.

${ }^{\circledR} 2019$ Hultén MA. This article is distributed under the terms of the Creative Commons Attribution 4.0 International License.
It is well known that most Down Syndrome cases are of maternal origin, and that the likelihood of having a child with Trisomy 21 increases with maternal age. We have now tested the possibility that the extra chromosome in Trisomy 21 Down syndrome may be due to a segregation problem, taking place during fetal mitotic oogonial cell divisions. In this context, we have analysed the chromosome constitution in 27150 pre-meiotic oogonia from 26 fetal ovarian samples with FISH technique and found that the incidence of Trisomy 21 cell nuclei is raised at later stages of fetal oogonial development. This feature can possibly explain the maternal age effect.

Down Syndrome (DS) is the most common condition associated with intellectual disability, and likely the only one readily recognised by most people in the general population. The clinical picture was first described in 1866 by the British physician John Langdon Down. Since then much effort has been devoted to the understanding of DS etiology. In 1959 it was discovered that DS is caused by an extra copy of chromosome 21, Trisomy 21. It is now well known that most DS cases are of maternal origin, and that the likelihood of having a child with a Trisomy 21 increases with maternal age ${ }^{1}$.

In sharp contrast, the underlying mechanism and origin of the extra chromosome 21 in DS has been a matter of debate for a long time. It has become generally accepted that the major cause is a faulty maternal reductional/meiotic cell division that occurs postpuberty at the monthly ovulation ${ }^{2}$. An inherent problem in this meiotic cell division is suggested to lead to the oocyte obtaining an extra chromosome 21, thus containing 24 chromosomes in total rather than the normal 23.

Our research group has instead focused attention on a totally different process, giving rise to oocytes with 24 chromosomes. We have tested the possibility that the extra chromosome 21 may be due to a segregation problem, taking place much earlier than postpuberty meiosis, i.e. during fetal mitotic oogonial cell divisions.

We have now analyzed the chromosome constitution in 27150 premeiotic oogonia from ovarian samples of 26 different fetuses ${ }^{3}$. The result of the chromosome analysis is illustrated in the Figure 1 , where data from eight, previously unpublished cases, are marked by their Reference Numbers. It is seen that the incidence of Trisomy 21 cell nuclei is raised at later stages of fetal oogonial development. This feature can explain a maternal age effect, since mammalian eggs are ovulated in a "first-in-first-out" order". In cases of fetal T21 there is a substantial delay in fetal oocyte maturation in comparison to that seen in cases with a normal karyotype ${ }^{5}$. In 


\section{T21 germ cells in fetal ovaries}

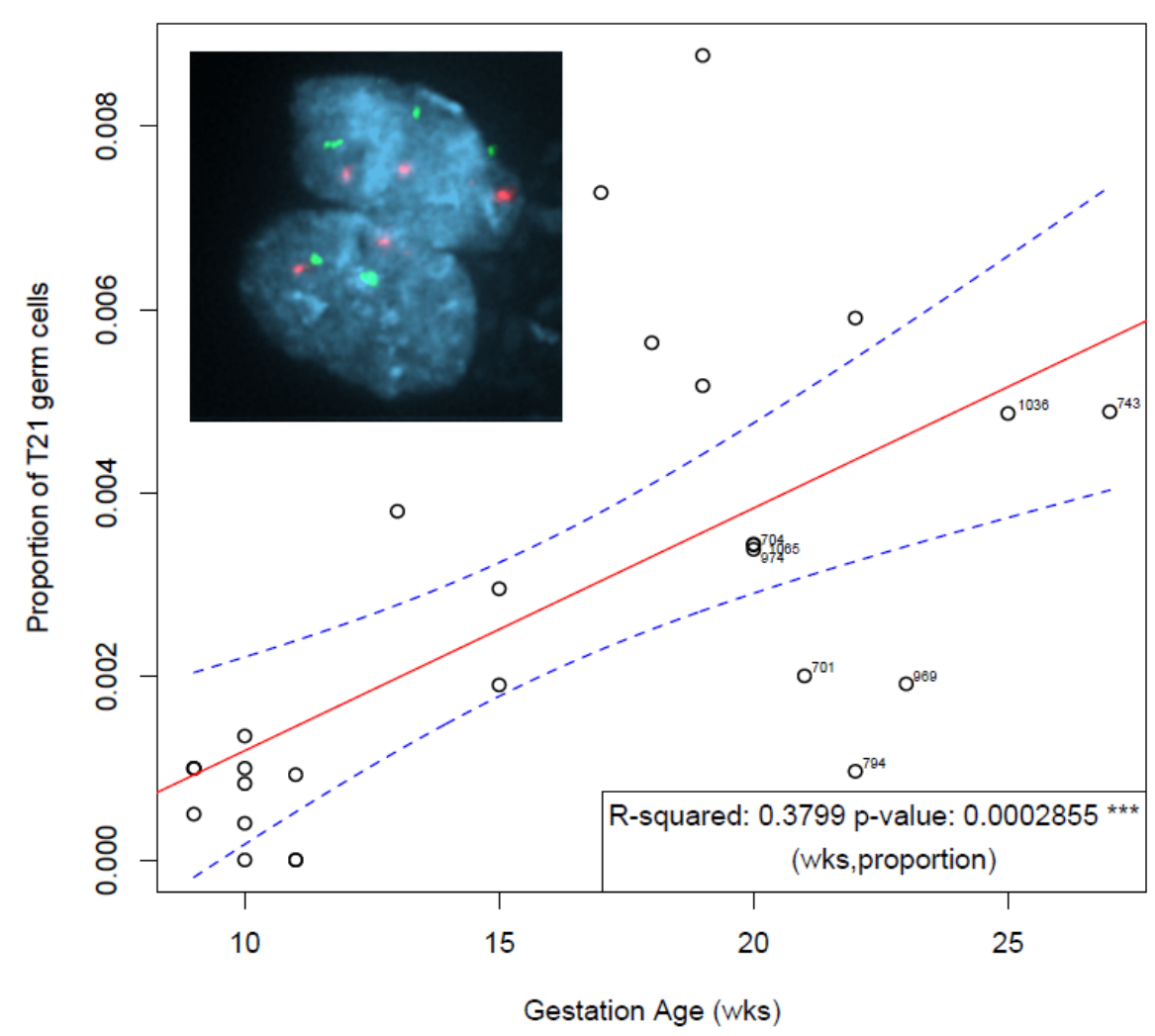

Figure 1: The type of chromosome analysis used in this study is illustrated by the two cell nuclei, where the bottom one has two redgreen fluorescence markers, corresponding to the two chromosomes 21 in a normal nucleus. On the other hand, the top nucleus has three red-green markers and has therefore been classified as having three chromosomes 21, Trisomy 21. In view of the complete absence of triploid cell nuclei in our previous series, we omitted the use of a complementary chromosome 18-specific probe as a control to differentiate between true T21 cell nuclei and triploid cell nuclei when analysing the eight additional samples.

The graph shows the result of the chromosome analysis, including fetal ovaries at the different gestational ages from 9-27 weeks, where the eight cases not published previously, are marked by their Reference Numbers. It is seen that the incidence of T21 nuclei is raised by gestational age.

all likelihood, the maternal age effect is augmented by the delayed progression of trisomic oocytes through meiotic prophase, resulting in their accumulation and ovulation later in reproductive life.

Substantial statistical support for the mitotic oogonial etiology of T21 has recently been presented by Horányi et $a l^{6}$, i.e. that women, who have used oral contraception pills at young age, have a reduced likelihood of begetting a child with DS at later reproductive age. The implication of this observation is the differentiation between the two alternatives for the etiology of T21 DS, invalidating the meiotic one, which is based on premature loss of centromeric cohesion as the major source of aneuploidy in oocytes from older women, because it is hardly conceivable that use of contraceptives at young age would prevent depletion of oocyte chromosomal cohesins in ageing ovaries.

Clinically, our study implies that therapeutic strategies to reduce the amount of trisomic germ cells are more or less out of reach, since the intervention must occur even before birth. Therefore, the present findings could be an argument for offering women, who want to postpone motherhood, egg freezing.

It is of further interest that T21 mosaicism, i.e. a proportion of T21 cell nuclei in different normal tissue samples is much more common than previously recognized ${ }^{7}$.

\section{References}

1. Penrose L. The relative effects of paternal and maternal age in mongolism. J Genet. 1933; 27: 219-224.

2. Herbert M, Kalleas D, Cooney D, et al. Meiosis and maternal aging: insights from aneuploid oocytes and trisomy births. Cold Spring Harb Perspect Biol. 2015; 7(4): a017970. Published. doi:10.1101/ cshperspect.a017970

3. Hultén MA, Öijerstedt L, Iwarsson E, et al. Maternal Germinal Trisomy 21 in Down Syndrome. J Clin Med. 2014; 3(1): 167-75. 
4. Polani PE, Crolla JA. A test of the production line hypothesis of mammalian oogenesis. Hum Genet. 1991 Nov; 88(1): 64-70.

5. Barlow AL, Tease C, Hultén MA. Meiotic chromosome pairing in fetal oocytes of trisomy 21 human females. Cytogenet Genome Res. 2002; 96: 45-51.

6. Horányi D, Babay LÉ, Rigó J Jr, et al. Effect of extended oral contraception use on the prevalence of fetal trisomy 21 in women aged at least 35 years. Int J Gynaecol Obstet. 2017; 138(3): 261-266. Erratum in: Int J Gynaecol Obstet. 2018; 140(2): 258.

7. Hultén MA, Jonasson J, Iwarsson E, et al. Trisomy 21 mosaicism. We may all have a touch of Down Syndrome: Cytogenet Genome Res. 2013; 139(3): 189-92. 